\title{
Weighted Markov-type inequalities, norms of Volterra operators, and zeros of Bessel functions
}

\author{
Albrecht Böttcher and Peter Dörfler \\ To the memory of Erhard Schmidt (1876-1959)
}

\begin{abstract}
The first term of the asymptotics of the best constants in Markov-type inequalities for higher derivatives of polynomials is determined in the two cases where the underlying norm is the $L^{2}$ norm with Laguerre weight or the $L^{2}$ norm with Gegenbauer weight. The coefficient in this term is shown to be the norm of a certain Volterra integral operator which depends on the weight and the order of the derivative. For first order derivatives, the norms of the Volterra operators are expressed in terms of the zeros of Bessel functions. The asymptotic behavior of the coefficients is studied and tight bounds for them are given.
\end{abstract}

Keywords: Markov-type inequality, orthogonal polynomials, Volterra integral operators, Bessel function, singular values

Mathematics Subject Classification (2000): Primary 41A10; Secondary 15A18, 26D10, 33A40, 41A44, 45D05, 47G10

\section{$1 \quad$ Introduction and main results}

An inequality of the Markov-type is an inequality of the form $\left\|f^{(\nu)}\right\| \leq C\|f\|$ for $f$ in the linear space $\mathcal{P}_{n}$ of all algebraic polynomials with complex coefficients of degree at most $n$. Here $f^{(\nu)}$ denotes the $\nu$ th derivative. The best possible constant $C$ depends on $n, \nu$, and the norm $\|\cdot\|$, and the determination and estimation of $C$ has been the subject of numerous investigations since Andrei Markov's paper [27] $\left(\nu=1\right.$ and $\|\cdot\|$ being the $L^{\infty}$ norm on a bounded interval) and paper [28] by his brother Vladimir Markov ( $\nu \geq 2$ and the same $L^{\infty}$ norm). We refer to [4], [19], [30], [31] for more on the early history and for recent developments.

This paper is devoted to the constant $C$ in the two cases where $\|\cdot\|$ is the $L^{2}$ norm with the Laguerre weight $t^{\alpha} e^{-t}$ on $(0, \infty)$ or the $L^{2}$ norm with the Gegenbauer (= ultraspherical) weight $\left(1-t^{2}\right)^{\alpha}$ on $(-1,1)$. Thus, we are interested in the best possible constants $\lambda_{n}^{(\nu)}$ such that

$$
\left\|f^{(\nu)}\right\| \leq \lambda_{n}^{(\nu)}\|f\| \quad \text { for all } \quad f \in \mathcal{P}_{n}
$$


where

$$
\|f\|^{2}=\int_{0}^{\infty}|f(t)|^{2} t^{\alpha} e^{-t} d t
$$

and in the best possible constants $\gamma_{n}^{(\nu)}$ for which

$$
\left\|f^{(\nu)}\right\| \leq \gamma_{n}^{(\nu)}\|f\| \quad \text { for all } \quad f \in \mathcal{P}_{n}
$$

provided

$$
\|f\|^{2}=\int_{-1}^{1}|f(t)|^{2}\left(1-t^{2}\right)^{\alpha} d t
$$

In both cases $\alpha>-1$ is a real number. We consider $\alpha$ as fixed and therefore suppress dependence on $\alpha$ in our notations.

We remark that, as already observed in [10], [33], [34], the problem is trivial for the Hermite weight $e^{-t^{2}}$ on $(-\infty, \infty)$. Indeed, in that case the normalized Hermite polynomials $h_{k}$ constitute an orthonormal basis $\left\{h_{0}, h_{1}, \ldots, h_{n}\right\}$ in $\mathcal{P}_{n}$ and the best possible constant $C$, which will be denoted by $\eta_{n}^{(\nu)}$, is the spectral norm of the matrix representation of the operator $f \mapsto f^{(\nu)}$ in this basis. The $k$ th column of this matrix consists of the coefficients in $h_{k}^{(\nu)}=c_{0, k} h_{0}+c_{1, k} h_{1}+\ldots+c_{k, k} h_{k}$, and since

$$
h_{k}^{(\nu)}=\sqrt{2^{\nu} \nu !\left(\begin{array}{l}
k \\
\nu
\end{array}\right)} h_{k-\nu}=\sqrt{2^{\nu} \frac{\Gamma(k+1)}{\Gamma(k-\nu+1)}} h_{k-\nu},
$$

the only nonzero entries of this matrix are on the $\nu$ th superdiagonal, the maximum modulus of these entries being

$$
\eta_{n}^{(\nu)}=\sqrt{2^{\nu} \frac{\Gamma(n+1)}{\Gamma(n-\nu+1)}}
$$

for $1 \leq \nu \leq n$. In particular, $\lim _{n \rightarrow \infty} \eta_{n}^{(\nu)} / n^{\nu / 2}=2^{\nu / 2}$.

In 1932, Erhard Schmidt [32] considered the case of the Legendre weight (that is, the Gegenbauer weight with $\alpha=0$ ) and discovered that $\gamma_{n}^{(1)} / n^{2} \rightarrow 1 / \pi$. Hille, Szegö, and Tamarkin [21] studied the Legendre case with the norm (4) replaced by its $L^{p}$ analogue and also obtained that $\gamma_{n}^{(1)} / n^{2} \rightarrow 1 / \pi$ for $p=2$, mentioning that this result was already announced without proof in [32]. In 1944, Schmidt [33] derived, again under the assumption that $\alpha=0$, the sharper asymptotic formulas

$$
\lambda_{n}^{(1)}=\frac{2 n+1}{\pi}\left(1-\frac{\pi^{2}}{24(2 n+1)^{2}}+\frac{R_{n}}{(2 n+1)^{4}}\right)^{-1}
$$

with $-8 / 3<R_{n}<4 / 3$ for $n \geq 2$ and

$$
\gamma_{n}^{(1)}=\frac{(n+3 / 2)^{2}}{\pi}\left(1-\frac{\pi^{2}-3}{12(n+3 / 2)^{2}}+\frac{R_{n}}{(n+3 / 2)^{4}}\right)^{-1}
$$


with $-6<R_{n}<13$ for $n \geq 5$. Thus, for $\alpha=0$ we have

$$
\lim _{n \rightarrow \infty} \frac{\lambda_{n}^{(1)}}{n}=\frac{2}{\pi}, \quad \lim _{n \rightarrow \infty} \frac{\gamma_{n}^{(1)}}{n^{2}}=\frac{1}{\pi} .
$$

Subsequently, Turán [39] showed that in the case $\alpha=0$ the exact value of $\lambda_{n}^{(1)}$ is

$$
\lambda_{n}^{(1)}=\left(2 \sin \frac{\pi}{4 n+2}\right)^{-1}
$$

for all $n \geq 1$. Shampine [34] studied second derivatives and established the estimate

$$
\frac{n^{2}}{k_{0}^{2}} \sqrt{1-\frac{k_{0}^{4}}{2 n}+\frac{k_{0}^{4}}{6 n^{2}}} \leq \lambda_{n}^{(2)} \leq \frac{n^{2}}{k_{0}^{2}}
$$

for $\alpha=0$, where $k_{0}=1.8751041 \ldots$ is the smallest positive root of the equation $1+\cos k \cosh k=0$. Clearly, this implies that $\lambda_{n}^{(2)} / n^{2} \rightarrow 1 / k_{0}^{2}$. Shampine also proved that $\gamma_{n}^{(2)} / n^{4} \rightarrow 1 /\left(4 k_{0}^{2}\right)$ for $\alpha=0$. Papers [11], [12], [14] are concerned with $\lambda_{n}^{(\nu)}$ if either $\nu$ or $\alpha$ are arbitrary. In [11] it is shown that if $\alpha=0$, then

$$
\frac{1}{2 \Gamma(\nu+1)} \sqrt{\frac{4}{2 \nu+1}} \leq \liminf _{n \rightarrow \infty} \frac{\lambda_{n}^{(\nu)}}{n^{\nu}} \leq \limsup _{n \rightarrow \infty} \frac{\lambda_{n}^{(\nu)}}{n^{\nu}} \leq \frac{1}{2 \Gamma(\nu+1)} \sqrt{\frac{2 \nu}{2 \nu-1}}
$$

paper [12] contains the bounds

$$
\frac{1}{\sqrt{(\alpha+1)(\alpha+3)}} \leq \liminf _{n \rightarrow \infty} \frac{\lambda_{n}^{(1)}}{n} \leq \limsup _{n \rightarrow \infty} \frac{\lambda_{n}^{(1)}}{n} \leq \frac{1}{\sqrt{2(\alpha+1)}}
$$

for arbitrary $\alpha>-1$, and the result of [14] is that $\lambda_{n}^{(1)} / n$ converges to the inverse of the smallest positive zero of the Bessel function $J_{(\alpha-1) / 2}$. We also want to mention paper [29] which deals with (1), (2) under the assumption that $f \in \mathcal{P}_{n}$ has exact degree $n$ and nonnegative real coefficients. Finally, see the very recent paper [24] by Kroó for the exact value of $\gamma_{n}^{(1)}$ in the case $\alpha=0$.

Here are our main results.

Theorem 1.1 We have

$$
\lim _{n \rightarrow \infty} \frac{\lambda_{n}^{(\nu)}}{n^{\nu}}=\left\|L_{\nu}\right\|_{\infty}, \quad \lim _{n \rightarrow \infty} \frac{\gamma_{n}^{(\nu)}}{n^{2 \nu}}=\left\|G_{\nu}\right\|_{\infty}
$$

where $L_{\nu}$ and $G_{\nu}$ are the Volterra integral operators on $L^{2}(0,1)$ that are defined by

$$
\begin{aligned}
& \left(L_{\nu} f\right)(x)=\frac{1}{\Gamma(\nu)} \int_{x}^{1} x^{\alpha / 2} y^{-\alpha / 2}(y-x)^{\nu-1} f(y) d y \\
& \left(G_{\nu} f\right)(x)=\frac{1}{2^{\nu-1} \Gamma(\nu)} \int_{x}^{1} x^{1 / 2+\alpha} y^{1 / 2-\alpha}\left(y^{2}-x^{2}\right)^{\nu-1} f(y) d y
\end{aligned}
$$

and $\|\cdot\|_{\infty}$ denotes the operator norm. 
Note that $\left\|L_{\nu}\right\|_{\infty}=\left\|L_{\nu}^{*}\right\|_{\infty}$ and $\left\|G_{\nu}\right\|_{\infty}=\left\|G_{\nu}^{*}\right\|_{\infty}$ where

$$
\begin{aligned}
& \left(L_{\nu}^{*} f\right)(x)=\frac{1}{\Gamma(\nu)} \int_{0}^{x} x^{-\alpha / 2} y^{\alpha / 2}(x-y)^{\nu-1} f(y) d y \\
& \left(G_{\nu}^{*} f\right)(x)=\frac{1}{2^{\nu-1} \Gamma(\nu)} \int_{0}^{x} x^{1 / 2-\alpha} y^{1 / 2+\alpha}\left(x^{2}-y^{2}\right)^{\nu-1} f(y) d y .
\end{aligned}
$$

For $\alpha=0$ and the weight (2), this theorem was established in [5], and in the case where $\alpha=0$ and $\nu \leq 2$, such a result is implicitly already in Shampine's papers [34], [35]. The message of Theorem 1.1 is the existence of the limits and the identification of the limits as the operator norms of explicitly given integral operators. The following result shows that, rather surprisingly, the Gegenbauer case can be completely reduced to the Laguerre case. In the form $\lambda_{n}^{(\nu)} / \gamma_{n}^{(\nu)} \sim$ $2^{\nu} / n^{\nu}$ as $n \rightarrow \infty$ and for $\alpha=0$, this result was already conjectured by Shampine $[34]$.

Theorem 1.2 The norms of $L_{\nu}$ and $G_{\nu}$ are related by the equality

$$
\left\|G_{\nu}\right\|_{\infty}=\frac{1}{2^{\nu}}\left\|L_{\nu}\right\|_{\infty}
$$

Finding explicit and useful exact expressions for the norms $\left\|L_{\nu}\right\|_{\infty}$ for general $\nu$ and $\alpha$ seems to be impossible. For $\alpha=0$, the problem is well known at least since [20] and has been studied by many authors. Numerical values, tight bounds, and the asymptotic formula $\left\|L_{\nu}\right\|_{\infty} \sim 1 /(2 \Gamma(\nu+1))(\nu \rightarrow \infty)$ can be found in [1], [5], [17], [18], [23], [25], [26], [38]. Paper [5] contains a sketch of the recent history of the subject. The notation $x_{\nu} \sim y_{\nu}$ means that $x_{\nu} / y_{\nu} \rightarrow 1$.

The following result gives a complete answer for $\nu=1$ and general $\alpha>-1$. For $\tau>-1$, let $J_{\tau}$ be the Bessel function

$$
J_{\tau}(x)=\sum_{k=0}^{\infty} \frac{(-1)^{k}}{\Gamma(k+1) \Gamma(k+1+\tau)}\left(\frac{x}{2}\right)^{2 k+\tau} .
$$

The emergence of eigenvalues of differential operators in connection with the singular values of integral operators is standard, and accordingly zeros of Bessel functions have occurred in this context in several places, in [2], [3], [14], [15], [16], [20] for example. In particular, in [14] it was shown that the limit of $\lambda_{n}^{(1)} / n$ is the inverse of the first positive zero of $J_{(\alpha-1) / 2}$. The method used there is different from the arguments we will employ here.

Theorem 1.3 A number $\mu>0$ is a singular value of the operator $L_{1}$ if and only if $J_{(\alpha-1) / 2}(1 / \mu)=0$ and a singular value of the operator $G_{1}$ if and only if $J_{(\alpha-1) / 2}(1 /(2 \mu))=0$. In particular, $\left\|L_{1}\right\|_{\infty}$ is the inverse of the smallest positive zero of $J_{(\alpha-1) / 2}$ and $\left\|G_{1}\right\|_{\infty}$ is half of the inverse of the first positive zero of $J_{(\alpha-1) / 2}$. 
Note that (5) follows from Theorems 1.1 and 1.3 for $\alpha=0$. For general $\nu \geq 1$ and $\alpha>-1,\left\|L_{\nu}\right\|_{\infty}$ can be shown to be the largest $\mu>0$ such that the differential equation

$$
(-1)^{\nu}\left(x^{-\alpha} g^{(\nu)}(x)\right)^{(\nu)}=\frac{1}{\mu^{2}} x^{-\alpha} g(x)
$$

with the boundary conditions

$$
g^{(j)}(0)=0 \text { and }\left.\left(x^{-\alpha} g^{(\nu)}(x)\right)^{(j)}\right|_{x=1}=0 \text { for } j=0,1, \ldots, \nu-1
$$

has a nontrivial solution. A function $g$ satisfies (6), (7) if and only if the function $f$ given by $f(x)=x^{-\alpha / 2} g(x)$ is an $L^{2}$ solution of the equation $L_{\nu}^{*} L_{\nu} f=\mu^{2} f$.

The first of the following two theorems gives asymptotically sharp bounds and hence also the asymptotics of the norms $\left\|L_{\nu}\right\|_{\infty}$ as $\nu \rightarrow \infty$, and the second provides us with even better bounds for these norms. We define numbers $C_{\nu}, \Gamma_{\nu}(\alpha), I_{\nu}(\alpha)$ by

$$
\begin{aligned}
& C_{\nu}=\frac{1}{\Gamma(\nu)}, \quad \Gamma_{\nu}(\alpha)^{2}=\frac{\Gamma(\alpha+1) \Gamma(2 \nu-1)}{\Gamma(\alpha+2 \nu)} \\
& I_{\nu}(\alpha)^{4}=\int_{0}^{1} x^{-\alpha} \int_{0}^{x} y^{-\alpha}\left(\int_{0}^{y} t^{\alpha}(x-t)^{\nu-1}(y-t)^{\nu-1} d t\right)^{2} d y d x .
\end{aligned}
$$

Theorem 1.4 For every $\alpha>-1$ and $\nu \geq 1$,

$$
\frac{C_{\nu} \Gamma_{\nu}(\alpha)}{\sqrt{2 \nu+\alpha+1}} \leq\left\|L_{\nu}\right\|_{\infty} \leq \frac{C_{\nu} \Gamma_{\nu}(\alpha)}{\sqrt{2 \nu}}
$$

In particular, as $\nu \rightarrow \infty$,

$$
\left\|L_{\nu}\right\|_{\infty} \sim \frac{C_{\nu} \Gamma_{\nu}(\alpha)}{\sqrt{2 \nu}} \sim \frac{\sqrt{\Gamma(\alpha+1)}}{2^{1+\alpha / 2}} \frac{1}{\Gamma(\nu) \nu^{1+\alpha / 2}} .
$$

Theorem 1.5 Suppose $\nu \geq 1$ and $\alpha>-1$. Let $k \geq 1$ be the integer given by

$$
k<\frac{\Gamma_{\nu}(\alpha)^{4}}{8 \nu^{2} I_{\nu}(\alpha)^{4}} \leq k+1
$$

Then

$$
\frac{C_{\nu}^{2} \Gamma_{\nu}(\alpha)^{2}}{2(k+1) \nu}\left(1+\sqrt{\frac{1}{k}\left(\frac{8(k+1) \nu^{2} I_{\nu}(\alpha)^{4}}{\Gamma_{\nu}(\alpha)^{4}}-1\right)}\right) \leq\left\|L_{\nu}\right\|_{\infty}^{2} \leq \sqrt{2} C_{\nu}^{2} I_{\nu}(\alpha)^{2}
$$

Theorem 1.5 is illustrated by three tables. In the first two tables, the indicated digits are correct because the two bounds coincide in these digits. The third table concerns the case $\alpha=100$ and shows the value of the number $k$ and the 


\begin{tabular}{|l|l|l|}
\hline & $\alpha=-99 / 100$ & $\alpha=-1 / 2$ \\
\hline$\nu=2$ & $4.962172 \ldots$ & $0.5128 \ldots$ \\
$\nu=3$ & $2.02007003 \ldots$ & $0.18357 \ldots$ \\
$\nu=4$ & $0.582089421 \ldots$ & $0.0486021 \ldots$ \\
$\nu=10$ & $6.055474637 \ldots \times 10^{-6}$ & $3.941630 \ldots \times 10^{-7}$ \\
$\nu=20$ & $1.272658121668 \ldots \times 10^{-17}$ & $6.9354560 \ldots \times 10^{-19}$ \\
$\nu=30$ & $1.426637958642 \ldots \times 10^{-31}$ & $7.0212160 \ldots \times 10^{-33}$ \\
\hline
\end{tabular}

The values of $\left\|L_{\nu}\right\|_{\infty}$ for several choices of $\nu$ and $\alpha$.

\begin{tabular}{|l|l|l|l|}
\hline & $\alpha=1 / 2$ & $\alpha=1$ & $\alpha=5$ \\
\hline$\nu=2$ & $0.190 \ldots$ & $0.139 \ldots$ & $0.034 \ldots$ \\
$\nu=3$ & $0.05502 \ldots$ & $0.0368 \ldots$ & $0.0055 \ldots$ \\
$\nu=4$ & $0.012512 \ldots$ & $0.00782 \ldots$ & $0.00077 \ldots$ \\
$\nu=10$ & $6.3093 \ldots \times 10^{-8}$ & $3.15873 \ldots \times 10^{-8}$ & $6.839 \ldots \times 10^{-10}$ \\
$\nu=20$ & $7.8023 \ldots \times 10^{-20}$ & $3.290333 \ldots \times 10^{-20}$ & $1.996116 \ldots \times 10^{-22}$ \\
$\nu=30$ & $6.4361031 \ldots \times 10^{-34}$ & $2.4538736 \ldots \times 10^{-34}$ & $6.882594 \ldots \times 10^{-37}$ \\
\hline
\end{tabular}

The values of $\left\|L_{\nu}\right\|_{\infty}$ for another set of choices of $\nu$ and $\alpha$.

\begin{tabular}{|l|l|l|}
\hline$\nu=1$ & $k=51$ & $0.0098<\left\|L_{\nu}\right\|_{\infty}<0.0263$ \\
$\nu=2$ & $k=10$ & $0.00020<\left\|L_{\nu}\right\|_{\infty}<0.00038$ \\
$\nu=3$ & $k=5$ & $4.013 \times 10^{-6}<\left\|L_{\nu}\right\|_{\infty}<6.040 \times 10^{-6}$ \\
$\nu=4$ & $k=3$ & $7.422 \times 10^{-8}<\left\|L_{\nu}\right\|_{\infty}<9.932 \times 10^{-8}$ \\
$\nu=10$ & $k=1$ & $1.803 \times 10^{-18}<\left\|L_{\nu}\right\|_{\infty}<1.832 \times 10^{-18}$ \\
$\nu=20$ & $k=1$ & $9.038 \times 10^{-37}<\left\|L_{\nu}\right\|_{\infty}<9.044 \times 10^{-37}$ \\
$\nu=30$ & $k=1$ & $1.24847 \times 10^{-55}<\left\|L_{\nu}\right\|_{\infty}<1.24858 \times 10^{-55}$ \\
\hline
\end{tabular}

The values of $k$ and the bounds delivered by Theorem 1.5 for $\alpha=100$.

two bounds from Theorem 1.5 for several values of $\nu$. For $\alpha=0$, numerical values for $\left\|L_{\nu}\right\|_{\infty}$ obtained on the basis of three completely different computational strategies are in [18], [38], [25], and [5]. Notice that the case $\nu=1$ is settled by Theorem 1.3.

This paper is a continuation of our paper [5]. The approach used in [5] and here is based on two ideas. The first is from [10] and consists in representing the operator $D^{\nu}: \mathcal{P}_{n} \rightarrow \mathcal{P}_{n}, f \mapsto f^{(\nu)}$ by a matrix in an orthogonal basis in $\mathcal{P}_{n}$ which is formed by orthogonal polynomials. The best constant in question is then just the operator norm and thus the maximal singular value of this matrix on $\mathbf{C}^{n+1}$ with the $\ell^{2}$ norm. Schmidt [33] and Shampine [34], [35] did not work with the matrix of the operator $D^{\nu}$ but rather with the maximal eigenvalue of the symmetric matrix representing $\left(D^{\nu}\right)^{*} D^{\nu}$. Since those symmetric matrices become 
quite complicated as $\nu$ increases, we have the limitation to $\nu=1$ and $\nu=2$ in [33], [34], [35].

The second idea goes back to Harold Widom [40], [41], [42] and independently again to Lawrence Shampine [34], [35]. They proposed that in order to study spectral properties of a sequence of $n \times n$ matrices one could replace the matrices by integral operators with piecewise constant kernels on $L^{2}(0,1)$ and then show that, after appropriate scaling, these integral operators converge to some limiting integral operator as $n$ goes to infinity. Interestingly, Lao and Whitley [25] proceeded in the reverse direction: they approximated Volterra integral operators by integral operators with piecewise constant kernels and thus by matrices and then numerically computed the norm of the matrices in order to get approximations for the norm of the integral operators. That we can go much further than Schmidt and Shampine is based on the exploitation of the fact that the replacement of matrices by integral operators is an algebraic homomorphism. Thus, we do not replace $\left(D^{\nu}\right)^{*} D^{\nu}$ by an integral operator but rather transform the sole $D$ into an integral operator $K_{n}$ and then simply prove that $K_{n} \rightarrow K$ in order to conclude that $\left(K_{n}^{\nu}\right)^{*} K_{n}^{\nu} \rightarrow\left(K^{\nu}\right)^{*} K^{\nu}$.

Finally, we should remark that in contrast to [5] (and [6] and [7]) the matrices we are encountering here are in general no longer Toeplitz.

The paper is organized as follows. In Sections 2 and 3 we derive the matrix representations of the operator of differentiation in orthogonal bases formed by Laguerre and Gegenbauer polynomials. In Section 4 we pass from matrices to integral operators and prove Theorems 1.1 and 1.2. Section 5 contains the proof of Theorem 1.3. In Section 6 we present the proof of Theorems 1.4 and 1.5.

We have not been able to find the asymptotics of the entries of the matrix representation of the operator of differentiation in Jacobi polynomials, that is, with the weight $(4)$ replaced by $(1-t)^{\alpha_{1}}(1+t)^{\alpha_{2}}$. The entries themselves were computed in [9]. However, the results of Sections 4 to 6 are all proved in a setting that is more general than necessary in order to dispose of the Laguerre and Gegenbauer cases. In this way we can treat these two cases simultaneously on the one hand, and if some day it will turn out that for Jacobi weights the asymptotic behavior of the entries is of the form (18), then our results will at once cover the Jacobi case on the other. In particular, the theorems proved in Sections 4 to 6 are more general than Theorems 1.1 to 1.5.

Acknowledgement. We thank Hermann Brunner for a useful discussion and his valuable hints to the literature on norms of Volterra operators. 


\section{Laguerre polynomials}

Fix $\alpha>-1$. The $k$ th Laguerre polynomial is

$$
R_{k}(t)=\frac{1}{\Gamma(k+1)} t^{-\alpha} e^{t} \frac{d^{k}}{d t^{k}}\left(t^{k+\alpha} e^{-t}\right)
$$

and the $k$ th normalized Laguerre polynomial is given by

$$
r_{k}(t)=\sqrt{\frac{\Gamma(k+1)}{\Gamma(k+\alpha+1)}} R_{k}(t) .
$$

The set $\mathcal{E}=\left\{r_{0}, r_{1}, \ldots, r_{n}\right\}$ is an orthonormal basis in $\mathcal{P}_{n}$ with the norm (2). Using the well known identity $R_{k}^{\prime}=-R_{0}-R_{1}-\ldots-R_{k-1}$ we obtain that, for $0 \leq k \leq n$,

$$
r_{k}^{\prime}=\sum_{j=0}^{k-1} c_{j k} r_{j} \quad \text { with } \quad c_{j k}=-\sqrt{\frac{\Gamma(j+\alpha+1)}{\Gamma(j+1)} \frac{\Gamma(k+1)}{\Gamma(k+\alpha+1)}} .
$$

Consequently, the operator $\mathcal{P}_{n} \rightarrow \mathcal{P}_{n}, f \mapsto f^{\prime}$ is in the basis $\mathcal{E}$ represented by the matrix

$$
D_{n+1}=\left(\begin{array}{rrrrr}
0 & c_{01} & c_{02} & \ldots & c_{0 n} \\
& 0 & c_{12} & \ldots & c_{1 n} \\
& & & & \ldots \\
& & & & c_{n-1, n} \\
& & & & 0
\end{array}\right) .
$$

The best constant $\lambda_{n}^{(\nu)}$ in (1) is therefore just the norm of $D_{n+1}^{\nu}$ as an operator on $\mathbf{C}^{n+1}$ with the $\ell^{2}$ norm. We may clearly ignore the minus sign in (10), and hence we consider

$$
A_{n+1}=\left(a_{j k}\right)_{j, k=0}^{n} \quad \text { with } \quad a_{j k}=-c_{j k}
$$

instead of $D_{n+1}$. Stirling's formula implies that if $\xi$ and $\eta$ are real numbers, then

$$
\frac{\Gamma(m+\xi)}{\Gamma(m+\eta)}=m^{\xi-\eta}(1+O(1 / m)) \quad \text { as } \quad m \rightarrow \infty .
$$

Thus, for the nonzero entries of $A_{n+1}$ we have

$$
a_{j k}=j^{\alpha / 2} k^{-\alpha / 2}(1+O(1 / j))(1+O(1 / k)) .
$$




\section{Gegenbauer polynomials}

Fix $\alpha>-1$ and consider $\mathcal{P}_{n}$ with the norm (4). This time an orthonormal basis $\mathcal{F}=\left\{p_{0}, p_{1}, \ldots, p_{n}\right\}$ in $\mathcal{P}_{n}$ is given by the normalized Gegenbauer polynomials

$$
p_{k}(t)=\sqrt{\frac{(2 k+2 \alpha+1) \Gamma(k+1) \Gamma(k+2 \alpha+1)}{2^{2 \alpha+1} \Gamma(k+\alpha+1)^{2}}} P_{k}(t)
$$

where $P_{k}$ is the polynomial

$$
P_{k}(t)=\frac{(-1)^{k}}{2^{k} \Gamma(k+1)}\left(1-t^{2}\right)^{-\alpha} \frac{d^{k}}{d t^{k}}\left[\left(1-t^{2}\right)^{k+\alpha}\right] .
$$

Finding the coefficients $c_{j k}$ in the decomposition

$$
p_{k}^{\prime}=\sum_{j=0}^{k-1} c_{j k} p_{j}
$$

is not as easy as in the Laguerre case. This computation can be found in [8] or [9], where it was shown that $c_{j k}=0$ if $j+k$ is even and

$$
c_{j k}=\sqrt{(2 j+2 \alpha+1)(2 k+2 \alpha+1)} \sqrt{\frac{\Gamma(j+2 \alpha+1)}{\Gamma(j+1)} \frac{\Gamma(k+1)}{\Gamma(k+2 \alpha+1)}}
$$

if $j+k$ is odd. With these $c_{j k}$ 's, the matrix $D_{n+1}$ representing the operator of differentiation in the basis $\mathcal{F}$ is of the form (11). Again the operator norm of $D_{n+1}^{\nu}$ coincides with the best possible constant $\gamma_{n}^{(\nu)}$ in (3).

To come up with the complications caused by the chessboard structure of $D_{n+1}$, we assume that $n$ is odd and put $n+1=2 m$. Note that, obviously, $\gamma_{n-1}^{(\nu)} \leq \gamma_{n}^{(\nu)} \leq \gamma_{n+1}^{(\nu)}$ for all $n$, so that it suffices to prove Theorem 1.1 in the case where the limit is taken over odd $n$ only. The chessboard matrix $D_{n+1}$ is unitarily equivalent through permutation matrices to the matrix

$$
\widetilde{D}_{n+1}=\left(\begin{array}{ll}
0 & A_{m} \\
B_{m} & 0
\end{array}\right)
$$

where $A_{m}$ and $B_{m}$ are the $m \times m$ matrices

$$
\left(\begin{array}{llll}
c_{01} & c_{03} & \ldots & c_{0,2 m-1} \\
& c_{23} & \ldots & c_{2,2 m-1} \\
& & \ddots & \vdots \\
& & & c_{2 m-2,2 m-1}
\end{array}\right), \quad\left(\begin{array}{lllll}
0 & c_{12} & c_{14} & \ldots & c_{1,2 m-2} \\
& 0 & c_{34} & \ldots & c_{3,2 m-2} \\
& & \ddots & & \vdots \\
& & & \ddots & c_{2 m-3,2 m-2} \\
& & & & 0
\end{array}\right),
$$


respectively. It follows that $D_{n+1}^{2}, D_{n+1}^{3}, D_{n+1}^{4}, \ldots$ are unitarily equivalent to

$$
\begin{aligned}
& \left(\begin{array}{cc}
A_{m} B_{m} & 0 \\
0 & B_{m} A_{m}
\end{array}\right), \quad\left(\begin{array}{cc}
0 & A_{m} B_{m} A_{m} \\
B_{m} A_{m} B_{m} & 0
\end{array}\right), \\
& \left(\begin{array}{cc}
A_{m} B_{m} A_{m} B_{m} & 0 \\
0 & B_{m} A_{m} B_{m} A_{m}
\end{array}\right), \quad \ldots .
\end{aligned}
$$

The operator norms of these matrices are the maximum of the operator norms of the two nonzero blocks.

Writing $A_{m}=\left(a_{j k}\right)_{j, k=0}^{m-1}$ and $B_{m}=\left(b_{j k}\right)_{j, k=0}^{m-1}$ we have

$$
a_{j k}=c_{2 j, 2 k+1}, \quad b_{j k}=c_{2 j+1,2 k} .
$$

From (12) and (14) we therefore obtain that

$$
a_{j k}=4 j^{1 / 2+\alpha} k^{1 / 2-\alpha}(1+O(1 / j))(1+O(1 / k))
$$

for the nonzero entries of $A_{m}$ and that the nonzero entries $b_{j k}$ are also equal to the right-hand side of (16).

\section{From matrices to integral operators}

Our task is to find the asymptotic behavior of the norms of products of uppertriangular $N \times N$ matrices $A_{N}$ whose entries are of the form (13) and (16). As already said, we tackle this problem by having recourse to an idea by Widom [40], [41], [42] and Shampine [34], [35], which consists in replacing the matrices by integral operators with piecewise constant kernels and subsequently analyzing whether the sequence of integral operators converges to a limiting integral operator. This approach was also pursued in [5], [6], [7].

The operator norm $\|A\|_{\infty}$ of a matrix or an operator $A$ is defined as the supremum of $\|A x\|$ over all $x$ of norm 1 . All concrete operators $A$ we are encountering in this paper are compact operators on a separable Hilbert space and hence their operator norm is simply the largest singular value, that is, the positive square root of the largest eigenvalue of $A^{*} A$, where $A^{*}$ stands for the adjoint operator. In linear algebra, the operator norm is called the spectral norm. A compact operator $A$ on a separable Hilbert space is said to be a Hilbert-Schmidt operator if $\sum_{\ell} \mu_{\ell}^{2}$, the sum of the squares of its singular values counted with algebraic multiplicity, is finite, in which case the Hilbert-Schmidt norm is defined by $\|A\|_{2}^{2}=\sum_{\ell} \mu_{\ell}^{2}$. Operators induced by finite matrices $A=\left(a_{j k}\right)$ are always Hilbert-Schmidt, their Hilbert-Schmidt norm is frequently referred to as the Frobenius norm, and we have $\|A\|_{2}^{2}=\sum_{j, k}\left|a_{j k}\right|^{2}$. If $A$ is an integral operator on $L^{2}(0,1)$ of the form $(A f)(x)=\int_{0}^{1} k(x, y) f(y) d y$, then $A$ is Hilbert-Schmidt if and only if

$$
\int_{0}^{1} \int_{0}^{1}|k(x, y)|^{2} d x d y<\infty .
$$


If the number (17) is finite, it coincides with $\|A\|_{2}^{2}$. Let finally $\mathcal{B}\left(L^{2}(0,1)\right)$ denote the $C^{*}$-algebra of all bounded linear operators on $L^{2}(0,1)$.

Lemma 4.1 (Widom and Shampine) For $A=\left(a_{j k}\right)_{j, k=0}^{N-1} \in \mathbf{C}^{N \times N}$, let $W_{A}$ be the integral operator on $L^{2}(0,1)$ whose kernel is $k(x, y)=N a_{[N x],[N y]}$, where $[\cdot]$ denotes the integral part. Then the map

$$
W: \mathbf{C}^{N \times N} \rightarrow \mathcal{B}\left(L^{2}(0,1)\right), \quad A \mapsto W_{A}
$$

is an algebraic homomorphism which preserves both operator norms and HilbertSchmidt norms.

Proof. Let $I_{k}$ be the interval $(k / N,(k+1) / N)$, denote by $\chi_{k}$ the characteristic function of $I_{k}$, and consider the operators

$$
\begin{array}{ll}
U: \mathbf{C}^{N} \rightarrow L^{2}(0,1), \quad\left\{x_{k}\right\}_{k=0}^{N-1} \mapsto \sqrt{N} \sum_{k=0}^{N-1} x_{k} \chi_{k}, \\
V: L^{2}(0,1) \rightarrow \mathbf{C}^{N}, \quad f \mapsto\left\{\sqrt{N} \int_{I_{k}} f(x) d x\right\}_{k=0}^{N-1} .
\end{array}
$$

It can be readily verified that $\|U\|_{\infty}=\|V\|_{\infty}=1$, that $V U$ is the identity operator on $\mathbf{C}^{N}$, and that $U A V=W_{A}$ for every $A \in \mathbf{C}^{N \times N}$. This implies that

$$
W_{\alpha A+\beta B}=\alpha W_{A}+\beta W_{B}, \quad W_{A B}=W_{A} W_{B}
$$

for all $A, B \in \mathbf{C}^{N \times N}$ and all $\alpha, \beta \in \mathbf{C}$. Since

$$
\begin{aligned}
& \left\|W_{A}\right\|_{\infty}=\|U A V\|_{\infty} \leq\|A\|_{\infty}=\left\|V W_{A} U\right\|_{\infty} \leq\left\|W_{A}\right\|_{\infty}, \\
& \left\|W_{A}\right\|_{2}=\|U A V\|_{2} \leq\|A\|_{2}=\left\|V W_{A} U\right\|_{2} \leq\left\|W_{A}\right\|_{2},
\end{aligned}
$$

it follows that $\|A\|_{\infty}=\left\|W_{A}\right\|_{\infty}$ and $\left\|W_{A}\right\|_{2}=\|A\|_{2}$ for all $A \in \mathbf{C}^{N \times N}$.

Let $a_{j k}(j \geq 0, k \geq 0)$ be complex numbers such that

$$
a_{j k}= \begin{cases}j^{\delta} k^{\beta}(1+O(1 / j))(1+O(1 / k)) & \text { for } j \leq k \\ 0 & \text { for } j>k\end{cases}
$$

where $\delta$ and $\beta$ are real numbers. Here $O(1 / j)$ stands for a family of numbers $b_{j k}$ $(0 \leq j \leq k)$ such that $\left|b_{j k}\right| \leq C / j$ for all $j, k \geq 1$ with some constant $C$ that is independent of $j$ and $k$. The term $O(1 / k)$ has an analogous meaning. Put $A_{N}=\left(a_{j k}\right)_{j, k=0}^{N-1}$ and denote by $T$ the integral operator over $(0,1)$ with the kernel

$$
\varrho(x, y)=\left\{\begin{array}{lll}
x^{\delta} y^{\beta} & \text { for } \quad 0<x<y \\
0 & \text { for } \quad 0<y<x
\end{array}\right.
$$


Theorem 4.2 If $2 \delta+1>0$ and $\sigma:=\beta+\delta+1>0$, then $T$ is a Hilbert-Schmidt operator on $L^{2}(0,1)$ and

$$
\left\|N^{-\sigma} W_{A_{N}}-T\right\|_{2} \rightarrow 0 \quad \text { as } \quad N \rightarrow \infty .
$$

Proof. We have

$$
\|T\|_{2}^{2}=\int_{0}^{1} \int_{0}^{y} x^{2 \delta} y^{2 \beta} d x d y=\frac{1}{2 \sigma(2 \delta+1)}<\infty,
$$

which shows that $T$ is Hilbert-Schmidt. To prove (20), we divide the square $(0,1)^{2}$ into the $N^{2}$ squares

$$
Q_{j k}=\left(\frac{j}{N}, \frac{j+1}{N}\right) \times\left(\frac{k}{N}, \frac{k+1}{N}\right) \quad(j, k=0, \ldots, N-1) .
$$

Then

$$
\left\|N^{-\sigma} W_{A_{N}}-T\right\|_{2}^{2}=\sum_{j, k=0}^{N-1} \iint_{Q_{j k}}\left|\frac{a_{j k}}{N^{\beta+\delta}}-\varrho(x, y)\right|^{2} d x d y,
$$

the integrals on the right-hand side of (22) being zero for $j>k$. We first check the right-hand side of (22) along the boundary of the triangle $0<x<y<1$ and subsequently inside this triangle. In what follows we will repeatedly use the inequality $|a-\varrho|^{2} \leq 2\left(|a|^{2}+|\varrho|^{2}\right)$.

Let first $j=k=0$. Taking into account that

$$
\begin{aligned}
& \iint_{Q_{00}}\left|\frac{a_{00}}{N^{\beta+\delta}}\right|^{2} d x d y=\frac{\left|a_{00}\right|^{2}}{N^{2(\beta+\delta)}} \frac{1}{N^{2}}=\frac{\left|a_{00}\right|^{2}}{N^{2 \sigma}}, \\
& \iint_{Q_{00}}|\varrho(x, y)|^{2} d x d y=\int_{0}^{1 / N} \int_{0}^{y} x^{2 \delta} y^{2 \beta} d x d y=\frac{1}{2 \sigma(2 \delta+1)} \frac{1}{N^{2 \sigma}},
\end{aligned}
$$

we see that the $j=k=0$ term on the right of (22) goes to zero. We now consider the terms with $j=0$ and $k \geq 1$. We have

$$
\sum_{k=1}^{N-1} \iint_{Q_{0 k}}\left|\frac{a_{0 k}}{N^{\beta+\delta}}\right|^{2} d x d y=\sum_{k=1}^{N-1} \frac{O\left(k^{2 \beta}\right)}{N^{2(\beta+\delta)}} \frac{1}{N^{2}},
$$

and this is $O\left(1 / N^{2 \delta+1}\right)$ for $\beta \neq-1 / 2$ and $O\left((\log N) / N^{2 \sigma}\right)$ for $\beta=-1 / 2$. Furthermore,

$$
\begin{aligned}
& \sum_{k=1}^{N-1} \iint_{Q_{0 k}}|\varrho(x, y)|^{2} d x d y=\int_{1 / N}^{1} \int_{0}^{1 / N}|\varrho(x, y)|^{2} d x d y \\
& =\int_{1 / N}^{1} \int_{0}^{1 / N} x^{2 \delta} y^{2 \beta} d x d y=\frac{1}{2 \delta+1} \frac{1}{N^{2 \delta+1}} \int_{1 / N}^{1} y^{2 \beta} d y
\end{aligned}
$$


which is $O\left(1 / N^{2 \delta+1}\right)+O\left(1 / N^{2 \sigma}\right)$ for $\beta \neq-1 / 2$ and $O\left((\log N) / N^{2 \delta+1}\right)$ for $\beta=$ $-1 / 2$. Thus, the sum over the terms $0, k$ with $1 \leq k \leq N-1$ also goes to zero. We next turn to the terms with $j=k \geq 1$. Clearly,

$$
\sum_{k=1}^{N-1} \iint_{Q_{k k}}\left|\frac{a_{k k}}{N^{\beta+\delta}}\right|^{2} d x d y=O\left(\sum_{k=1}^{N-1} \frac{k^{2 \beta+2 \delta}}{N^{2(\beta+\delta)}} \frac{1}{N^{2}}\right)=O\left(\frac{1}{N}\right) .
$$

For the corresponding term with $|\varrho|^{2}$ we get

$$
\begin{aligned}
& \sum_{k=1}^{N-1} \iint_{Q_{k k}}|\varrho(x, y)|^{2} d x d y \leq \sum_{k=1}^{N-1} \int_{k / N}^{(k+1) / N} \int_{k / N}^{(k+1) / N} x^{2 \delta} y^{2 \beta} d x d y \\
& =\sum_{k=1}^{N-1}\left(\frac{k+\xi_{k}}{N}\right)^{2 \delta}\left(\frac{k+\eta_{k}}{N}\right)^{2 \beta} \frac{1}{N^{2}}
\end{aligned}
$$

with $0 \leq \xi_{k} \leq 1$ and $0 \leq \eta_{k} \leq 1$. It follows that (23) is at most

$$
\sum_{k=1}^{N-1} \frac{k^{2 \delta} k^{2 \beta}}{N^{2 \delta+2 \beta}} \frac{1}{N^{2}}\left(1+O\left(\frac{1}{k}\right)\right)=O\left(\sum_{k=1}^{N-1} \frac{k^{2 \beta+2 \delta}}{N^{2 \sigma}}\right),
$$

which is $O(1 / N)$ for $\beta+\delta \neq-1 / 2$ and $O\left((\log N) / N^{2 \sigma}\right)$ for $\beta+\delta=-1 / 2$. Consequently, the sum of the $k, k$ terms on the right of (22) goes to zero, too. It remains to consider the terms with $1 \leq j<k \leq N-1$. The sum of these terms is

$$
\sum_{j=1}^{N-1} \sum_{k=j+1}^{N-1} \int_{0}^{1} \int_{0}^{1}\left|\frac{a_{j k}}{N^{\beta+\delta}}-\varrho\left(\frac{j+x}{N}, \frac{k+y}{N}\right)\right|^{2} d x d y .
$$

For $0<x, y<1$,

$$
\begin{aligned}
& \varrho\left(\frac{j+x}{N}, \frac{k+y}{N}\right)=\left(\frac{j+x}{N}\right)^{\delta}\left(\frac{k+y}{N}\right)^{\beta} \\
& =\frac{j^{\delta} k^{\beta}}{N^{\beta+\delta}}\left(1+O\left(\frac{1}{j}\right)\right)\left(1+O\left(\frac{1}{k}\right)\right)=\frac{j^{\delta} k^{\beta}}{N^{\beta+\delta}}\left(1+O\left(\frac{1}{j}\right)+O\left(\frac{1}{k}\right)\right) .
\end{aligned}
$$

This and (18) yield that

$$
\begin{aligned}
& \left|\frac{a_{j k}}{N^{\beta+\delta}}-\varrho\left(\frac{j+x}{N}, \frac{k+y}{N}\right)\right|^{2}=\frac{j^{2 \delta} k^{2 \beta}}{N^{2(\beta+\delta)}}\left(O\left(\frac{1}{j}\right)+O\left(\frac{1}{k}\right)\right)^{2} \\
& =\frac{j^{2 \delta} k^{2 \beta}}{N^{2(\beta+\delta)}}\left(O\left(\frac{1}{j^{2}}\right)+O\left(\frac{1}{k^{2}}\right)\right) .
\end{aligned}
$$

Thus, (24) is at most a constant times

$$
\begin{aligned}
& \frac{1}{N^{2(\beta+\delta)}}\left(\sum_{j=1}^{N-1} j^{2 \delta-2} \sum_{k=j+1}^{N-1} k^{2 \beta}+\sum_{j=1}^{N-1} j^{2 \delta} \sum_{k=j+1}^{N-1} k^{2 \beta-2}\right) \frac{1}{N^{2}} \\
& \leq \frac{1}{N^{2 \sigma}} \sum_{j=1}^{N-1} j^{2 \delta-2} \sum_{k=1}^{N-1} k^{2 \beta}+\frac{1}{N^{2 \sigma}} \sum_{j=1}^{N-1} j^{2 \delta} \sum_{k=1}^{N-1} k^{2 \beta-2}=: S_{1}+S_{2} .
\end{aligned}
$$


It is easily seen that $S_{1}$ is $O\left(1 / N^{2}\right)$ if $\delta \neq 1 / 2$ and $\beta \neq-1 / 2$ and at most $O\left((\log N)^{2} / N^{2}\right)$ if $\delta=1 / 2$ or $\beta=-1 / 2$. The term $S_{2}$ is $O\left(1 / N^{2}\right)$ for $\beta \neq 1 / 2$ and $O\left((\log N) / N^{2}\right)$ for $\beta=1 / 2$.

Corollary 4.3 Let $A_{N}^{(\ell)}=\left(a_{j k}^{(\ell)}\right)_{j, k=0}^{N-1}(\ell=1, \ldots, \nu)$ be matrices whose entries satisfy

$$
a_{j k}^{(\ell)}= \begin{cases}j^{\delta} k^{\beta}(1+O(1 / j))(1+O(1 / k)) & \text { for } j \leq k, \\ 0 & \text { for } j>k .\end{cases}
$$

Put $\sigma:=\beta+\delta+1$ and suppose $\sigma>0$ and $2 \delta+1>0$. Denote by $T_{\nu}$ and $T_{\nu}^{*}$ the integral operators on $L^{2}(0,1)$ that are defined by

$$
\begin{aligned}
& \left(T_{\nu} f\right)(x)=\frac{1}{\sigma^{\nu-1} \Gamma(\nu)} \int_{x}^{1} x^{\delta} y^{\beta}\left(y^{\sigma}-x^{\sigma}\right)^{\nu-1} f(y) d y \\
& \left(T_{\nu}^{*} f\right)(x)=\frac{1}{\sigma^{\nu-1} \Gamma(\nu)} \int_{0}^{x} x^{\beta} y^{\delta}\left(x^{\sigma}-y^{\sigma}\right)^{\nu-1} f(y) d y .
\end{aligned}
$$

Then $T_{\nu}$ and $T_{\nu}^{*}$ are Hilbert-Schmidt operators and

$$
\lim _{N \rightarrow \infty} \frac{1}{N^{\sigma \nu}}\left\|A_{N}^{(1)} \ldots A_{N}^{(\nu)}\right\|_{\infty}=\left\|T_{\nu}\right\|_{\infty}=\left\|T_{\nu}^{*}\right\|_{\infty} .
$$

Proof. Since $\left|y^{\sigma}-x^{\sigma}\right| \leq 2$, we see from (21) that $T_{\nu}$ and thus also its adjoint operator $T_{\nu}^{*}$ are Hilbert-Schmidt. Theorem 4.2 shows that

$$
\left\|N^{-\sigma} W_{A_{N}^{(\ell)}}-T_{1}\right\|_{\infty} \leq\left\|N^{-\sigma} W_{A_{N}^{(\ell)}}-T_{1}\right\|_{2}=o(1)
$$

for each $\ell$, which together with Lemma 4.1 implies that

$$
\left\|N^{-\sigma \nu} W_{A_{N}^{(1)} \ldots A_{N}^{(\nu)}}-T_{1}^{\nu}\right\|_{\infty} \rightarrow 0 .
$$

Therefore the limit in (25) equals $\left\|T_{1}^{\nu}\right\|_{\infty}=\left\|\left(T_{1}^{*}\right)^{\nu}\right\|_{\infty}$. If $K$ is a Volterra integral operator of the form

$$
(K f)(x)=\int_{0}^{x} a(x) b(y) f(y) d y,
$$

then the $\nu$ th power $K^{\nu}$ is the Volterra integral operator given by

$$
\left(K^{\nu} f\right)(x)=\frac{1}{\Gamma(\nu)} \int_{0}^{x} a(x) b(y)\left(\int_{y}^{x} a(t) b(t) d t\right)^{\nu-1} f(y) d y .
$$

Letting $K=T_{1}^{*}, a(x)=x^{\beta}, b(y)=y^{\delta}$ we conclude that $\left(T_{1}^{*}\right)^{\nu}$ is nothing but the operator $T_{\nu}^{*}$ and that, consequently, $T_{1}^{\nu}=T_{\nu}$.

We are now in a position to prove Theorem 1.1. In the Laguerre case,

$$
\lambda_{n}^{(\nu)}=\left\|D_{n+1}^{\nu}\right\|_{\infty}=\left\|A_{n+1}^{\nu}\right\|_{\infty},
$$


and the entries of $A_{n+1}$ satisfy (13) for $j \leq k$. Corollary 4.3 with $N=n+1$, $\delta=\alpha / 2, \beta=-\alpha / 2, \sigma=1$ therefore immediately gives the assertion. Now consider the Gegenbauer case. As already said in Section 3, we may confine ourselves to odd $n$. We have

$$
\gamma_{n}^{(\nu)}=\left\|D_{n+1}^{\nu}\right\|_{\infty}=\left\|\widetilde{D}_{n+1}^{\nu}\right\|_{\infty},
$$

and the matrices $\widetilde{D}_{n+1}^{\nu}$ are given by (15). Taking into account that the entries of the matrices $A_{m}$ and $B_{m}$ satisfy (16), we deduce from Corollary 4.3 with $N=m=(n+1) / 2, \delta=1 / 2+\alpha, \beta=1 / 2-\alpha, \sigma=2$ that the operator norm of a product of the form $A_{m} B_{m} A_{m} \ldots$ or $B_{m} A_{m} B_{m} \ldots$ with $\nu$ factors is asymptotically equal to $4^{\nu}\left\|G_{\nu}\right\|_{\infty} m^{2 \nu} \sim\left\|G_{\nu}\right\|_{\infty} n^{2 \nu}$; note that in contrast to (18) there is the additional factor 4 in (16). As the operator norms of the matrices (15) are the maxima of the operator norms of their two nonzero blocks, it follows that $\left\|\widetilde{D}_{n+1}^{\nu}\right\|_{\infty} \sim\left\|G_{\nu}\right\|_{\infty} n^{2 \nu}$, as asserted.

Theorem 4.4 Suppose $2 \delta+1>0$ and $\sigma:=\beta+\delta+1>0$. Let $T_{\nu}$ be the operator given by

$$
\left(T_{\nu} f\right)(x)=\frac{1}{\sigma^{\nu-1} \Gamma(\nu)} \int_{x}^{1} x^{\delta} y^{\beta}\left(y^{\sigma}-x^{\sigma}\right)^{\nu-1} f(y) d y
$$

put $\alpha=(\delta-\beta) / \sigma$, and consider the operator

$$
\left(L_{\nu} f\right)(x)=\frac{1}{\Gamma(\nu)} \int_{x}^{1} x^{\alpha / 2} y^{-\alpha / 2}(y-x)^{\nu-1} f(y) d y .
$$

Then $\alpha>-1$ and the two operators $\sigma^{\nu} T_{\nu}$ and $L_{\nu}$ are unitarily equivalent.

Proof. It is clear that $\alpha>-1$. The operator given on $L^{2}(0,1)$ by

$$
(U f)(x)=\sigma^{1 / 2} x^{(\sigma-1) / 2} f\left(x^{\sigma}\right), \quad\left(U^{-1} f\right)(x)=\sigma^{-1 / 2} x^{(1-\sigma) /(2 \sigma)} f\left(x^{1 / \sigma}\right)
$$

is unitary. Denote the kernel of $L_{\nu}$ by $k(x, y)$. We have

$$
\begin{aligned}
\left(U L_{\nu} U^{-1} f\right)(x) & =\sigma^{1 / 2} x^{(\sigma-1) / 2} \int_{x^{\sigma}}^{1} k\left(x^{\sigma}, t\right)\left(U^{-1} f\right)(t) d t \\
& =\sigma^{1 / 2} x^{(\sigma-1) / 2} \int_{x}^{1} k\left(x^{\sigma}, y^{\sigma}\right)\left(U^{-1} f\right)\left(y^{\sigma}\right) \sigma y^{\sigma-1} d y \\
& =\sigma^{1 / 2} x^{(\sigma-1) / 2} \int_{x}^{1} k\left(x^{\sigma}, y^{\sigma}\right) \sigma^{-1 / 2} y^{(1-\sigma) / 2} f(y) \sigma y^{\sigma-1} d y \\
& =\sigma \int_{x}^{1} x^{(\sigma-1) / 2} y^{(\sigma-1) / 2} k\left(x^{\sigma}, y^{\sigma}\right) f(y) d y \\
& =\frac{\sigma}{\Gamma(\nu)} \int_{x}^{1} x^{\delta} y^{\beta}\left(y^{\sigma}-x^{\sigma}\right)^{\nu-1} f(y) d y=\sigma^{\nu}\left(T_{\nu} f\right)(x),
\end{aligned}
$$

which proves the assertion.

Theorem 4.4 with $\beta=1 / 2-\alpha$ and $\delta=1 / 2+\alpha$ immediately implies Theorem 1.2 . 


\section{Zeros of Bessel functions}

In this section we prove Theorem 1.3. The following theorem is not terribly new and the proof is based on standard arguments. For example, in the cases where $\beta=-1 / 2$ and $\delta=1 / 2$, the theorem is in [2], while under the additional assumption $2 \beta+1=2 \delta-1$ it can be found in [15] and [16]. For the reader's convenience, we give a full proof.

Theorem 5.1 Suppose $\beta$ and $\delta$ are real numbers satisfying $\sigma:=\beta+\delta+1>0$ and $2 \delta+1>0$. Then a number $\mu>0$ is a singular value of the operator $T_{1}$ defined on $L^{2}(0,1)$ by

$$
\left(T_{1} f\right)(x)=\int_{x}^{1} x^{\delta} y^{\beta} f(y) d y
$$

if and only if

$$
J_{-\frac{1+2 \beta}{2 \sigma}}\left(\frac{1}{\sigma \mu}\right)=0
$$

Proof. We have $\left(T_{1}^{*} f\right)(x)=\int_{0}^{x} x^{\beta} y^{\delta} f(y) d y$ and hence

$$
\begin{aligned}
\left(T_{1}^{*} T_{1} f\right)(x) & =\int_{0}^{x} x^{\beta} t^{\delta} \int_{t}^{1} t^{\delta} y^{\beta} f(y) d y d t \\
& =\int_{0}^{x} x^{\beta} \int_{0}^{y} t^{2 \delta} d t y^{\beta} f(y) d y+\int_{x}^{1} x^{\beta} \int_{0}^{x} t^{2 \delta} d t y^{\beta} f(y) d y \\
& =\frac{x^{\beta}}{2 \delta+1} \int_{0}^{x} y^{\beta+2 \delta+1} f(y) d y+\frac{x^{\beta+2 \delta+1}}{2 \delta+1} \int_{x}^{1} y^{\beta} f(y) d y .
\end{aligned}
$$

Let $\mu>0$ and suppose $\mu$ is a singular value of $T_{1}$. Then $T_{1}^{*} T_{1} f=\mu^{2} f$ for some nonzero $f \in L^{2}(0,1)$, that is,

$$
\int_{0}^{x} y^{\beta+2 \delta+1} f(y) d y+x^{2 \delta+1} \int_{x}^{1} y^{\beta} f(y) d y=(2 \delta+1) \mu^{2} x^{-\beta} f(x) .
$$

Put $g(x)=x^{-\beta} f(x)$. By assumption, $2 \delta+1>0$ and $2 \beta+4 \delta+3=2 \sigma+2 \delta+1>0$. Thus, if $\beta \neq-1 / 2$ then

$$
\begin{aligned}
x^{4 \delta+2}\left|\int_{x}^{1} y^{\beta} f(y) d y\right|^{2} & \leq x^{4 \delta+2} \int_{x}^{1} y^{2 \beta} d y \int_{0}^{1}|f(y)|^{2} d y \\
& =\frac{x^{4 \delta+2}-x^{2 \beta+4 \delta+3}}{2 \beta+1} \int_{0}^{1}|f(y)|^{2} d y=o(1)
\end{aligned}
$$

as $x \rightarrow 0$, while if $\beta=-1 / 2$ then

$$
\begin{aligned}
x^{4 \delta+2}\left|\int_{x}^{1} y^{-1 / 2} f(y) d y\right|^{2} & \leq x^{4 \delta+2} \int_{x}^{1} \frac{d y}{y} \int_{0}^{1}|f(y)|^{2} d y \\
& =x^{4 \delta+2}|\log x| \int_{0}^{1}|f(y)|^{2} d y=o(1) .
\end{aligned}
$$


Furthermore,

$$
\left|\int_{0}^{x} y^{\beta+2 \delta+1} f(y) d y\right|^{2} \leq \int_{0}^{x} y^{2 \beta+4 \delta+2} d y \int_{0}^{1}|f(y)|^{2} d y=o(1)
$$

as $x \rightarrow 0$. It follows that the left-hand side of (27) is an absolutely continuous function of $x$ that goes to zero as $x \rightarrow 0$. Consequently,

$$
g(0):=\lim _{x \rightarrow 0} g(x)=0
$$

We write (27) in the form

$$
\int_{0}^{x} y^{2 \sigma-1} g(y) d y+x^{2 \delta+1} \int_{x}^{1} y^{2 \beta} g(y) d y=(2 \delta+1) \mu^{2} g(x) .
$$

As in (29) all functions of $x$ are absolutely continuous, we may differentiate to get

$$
(2 \delta+1) x^{2 \delta} \int_{x}^{1} y^{2 \beta} g(y) d y=(2 \delta+1) \mu^{2} g^{\prime}(x) .
$$

The function $y^{2 \beta} g(y)=y^{\beta} f(y)$ belongs to $L^{1}(\varepsilon, 1)$ for each $\varepsilon>0$ and therefore $g^{\prime}(x)$ is absolutely continuous and satisfies

$$
g^{\prime}(1):=\lim _{x \rightarrow 1} g^{\prime}(x)=0
$$

Writing (30) in the form

$$
\int_{x}^{1} y^{2 \beta} g(y) d y=\mu^{2} x^{-2 \delta} g^{\prime}(x)
$$

and taking into account that both sides of this equality are absolutely continuous, we obtain after differentiation that

$$
-x^{2 \beta} g(x)=\mu^{2}(-2 \delta) x^{-2 \delta-1} g^{\prime}(x)+\mu^{2} x^{-2 \delta} g^{\prime \prime}(x)
$$

or equivalently,

$$
x^{2} g^{\prime \prime}(x)-2 \delta x g^{\prime}(x)+\frac{1}{\mu^{2}} x^{2 \sigma} g(x)=0 .
$$

In summary, if $f \in L^{2}(0,1)$ satisfies $(27)$, then $g(x)=x^{-\beta} f(x)$ is a solution of (32) with the boundary conditions (28) and (31).

The general solution of $(32)$ is known to be

$$
g(x)=x^{(1+2 \delta) / 2} Z_{\tau}\left(\frac{x^{\sigma}}{\sigma \mu}\right), \quad \tau:=\frac{2 \delta+1}{2 \sigma},
$$


where $Z_{\tau}=C_{1} J_{\tau}+C_{2} Y_{\tau}$ (see, e.g., [22, No. 2.163]). Because

$$
J_{\tau}(z) \sim \frac{1}{\Gamma(\tau+1)}\left(\frac{z}{2}\right)^{\tau}, \quad Y_{\tau}(z) \sim-\frac{\Gamma(\tau)}{\pi}\left(\frac{2}{z}\right)^{\tau}
$$

as $z \rightarrow 0$, condition (28) implies that $C_{2}=0$. We may therefore assume that $C_{1}=1$. It follows that

$$
g\left(x^{1 / \sigma}\right)=x^{(1+2 \delta) /(2 \sigma)} J_{\tau}\left(\frac{x}{\sigma \mu}\right)=x^{\tau} J_{\tau}\left(\frac{x}{\sigma \mu}\right) .
$$

From (31) we see that

$$
\left.\frac{d}{d x} g\left(x^{1 / \sigma}\right)\right|_{x=1}=\left.g^{\prime}\left(x^{1 / \sigma}\right) \frac{x^{1 / \sigma-1}}{\sigma}\right|_{x=1}=g^{\prime}(1) / \sigma=0 .
$$

Since $\left(z^{\tau} J_{\tau}(\alpha z)\right)^{\prime}=\alpha z^{\tau} J_{\tau-1}(\alpha z)$, we therefore obtain that

$$
0=\frac{d}{d x}\left[x^{\tau} J_{\tau}\left(\frac{x}{\sigma \mu}\right)\right]_{x=1}=\left.\frac{1}{\sigma \mu} x^{\tau} J_{\tau-1}\left(\frac{x}{\sigma \mu}\right)\right|_{x=1}=\frac{1}{\sigma \mu} J_{\tau-1}\left(\frac{1}{\sigma \mu}\right) .
$$

As $\tau-1=-(1+2 \beta) /(2 \sigma)$ we arrive at $(26)$.

Conversely, suppose (26) holds. Define $g(x)$ by (33) with $Z_{\tau}=J_{\tau}$ and put $f(x)=x^{\beta} g(x)$. We have

$$
\frac{d}{d x}\left[x^{\tau} J_{\tau}\left(\frac{x}{\sigma \mu}\right)\right]_{x=1}=\frac{1}{\sigma \mu} J_{\tau-1}\left(\frac{1}{\sigma \mu}\right)=0
$$

and hence

$$
g^{\prime}(1)=\left.\sigma \frac{d}{d x} g\left(x^{1 / \sigma}\right)\right|_{x=1}=0,
$$

that is, (31) holds. But from (32) and (31) we get (30). The first formula in (34) shows that $g(x)$ is asymptotically a constant times

$$
x^{(1+2 \delta) / 2} x^{\sigma \tau}=x^{(1+2 \delta) / 2} x^{(1+2 \delta) / 2}=x^{1+2 \delta}=o(1)
$$

as $x \rightarrow 0$ and that $x^{\beta} g(x)$ is asymptotically a constant times

$$
x^{\beta+1+2 \delta}=x^{\sigma+(2 \delta+1) / 2-1 / 2}
$$

as $x \rightarrow 0$. Thus, $g(0)=0$ and $f(x)=x^{\beta} g(x)$ is a function in $L^{2}(0,1)$. Since (30) and (28) give (27), we arrive at the conclusion that $f \in L^{2}(0,1)$ satisfies $T_{1}^{*} T_{1} f=\mu^{2} f$.

Corollary 5.2 If $\beta$ and $\delta$ are real numbers satisfying $\sigma:=\beta+\delta+1>0$ and $2 \delta+1>0$ and if $T_{1}$ is given by

$$
\left(T_{1} f\right)(x)=\int_{x}^{1} x^{\delta} y^{\beta} f(y) d y
$$

on $L^{2}(0,1)$, then $\left\|T_{1}\right\|_{\infty}=\left\|T_{1}^{*}\right\|_{\infty}$ equals $1 / \sigma$ times the inverse of the smallest positive zero of the function $J_{-(1+2 \beta) /(2 \sigma)}$. 
Proof. This is immediate from Theorem 5.1.

Theorem 1.3 is a straightforward consequence of Theorem 5.1 and Corollary 5.2: in the Laguerre case we have $\delta=\alpha / 2, \beta=-\alpha / 2, \sigma=1$ and in the Gegenbauer case the parameters are $\delta=1 / 2+\alpha, \beta=1 / 2-\alpha, \sigma=2$. Note that the part of Theorem 1.3 concerning the operator $G_{1}$ can also be derived from Theorem 5.1 and Corollary 5.2 for $\delta=\alpha / 2, \beta=-\alpha / 2, \sigma=1$ (Laguerre case) and Theorem 1.2.

Finally, by $2 \nu$ times differentiating the equation $L_{\nu}^{*} L_{\nu} f=\mu^{2} f$ and justifying the steps by arguments as in the proof of Theorem 5.1, one can derive equation (6) with the boundary conditions (7).

\section{Bounds for the norms of Volterra operators}

This section is devoted to the proofs of Theorems 1.4 and 1.5. Our approach follows [5] and [13].

Fix a real number $\alpha>-1$, let $C_{\nu}, \Gamma_{\nu}(\alpha), I_{\nu}(\alpha)$ be as in Section 1 , and abbreviate $\Gamma_{\nu}(\alpha)$ and $I_{\nu}(\alpha)$ to $\Gamma_{\nu}$ and $I_{\nu}$.

Lemma 6.1 We have

$$
\left\|L_{\nu}\right\|_{2}^{2}=\frac{C_{\nu}^{2} \Gamma_{\nu}^{2}}{2 \nu}, \quad\left\|L_{\nu}^{*} L_{\nu}\right\|_{2}^{2}=2 C_{\nu}^{4} I_{\nu}^{4} .
$$

Proof. Obviously,

$$
\begin{aligned}
& \left\|L_{\nu}\right\|_{2}^{2}=\left\|L_{\nu}^{*}\right\|_{2}^{2}=C_{\nu}^{2} \int_{0}^{1} \int_{0}^{x} x^{-\alpha} y^{\alpha}(x-y)^{2 \nu-2} d y d x \\
& =C_{\nu}^{2} \int_{0}^{1} x^{2 \nu-1} d x \int_{0}^{1} t^{\alpha}(1-t)^{2 \nu-2} d t=\frac{C_{\nu}^{2}}{2 \nu} \frac{\Gamma(\alpha+1) \Gamma(2 \nu-1)}{\Gamma(\alpha+2 \nu)}=\frac{C_{\nu}^{2} \Gamma_{\nu}^{2}}{2 \nu} .
\end{aligned}
$$

It can be verified straightforwardly that $\left(L_{\nu}^{*} L_{\nu} f\right)(x)=C_{\nu}^{2} \int_{0}^{1} c(x, y) f(y) d y$ where the symmetric kernel $c(x, y)$ is

$$
c(x, y)=x^{-\alpha / 2} y^{-\alpha / 2} \int_{0}^{y} t^{\alpha}(x-t)^{\nu-1}(y-t)^{\nu-1} d t
$$

for $y<x$. This implies that

$$
\left\|L_{\nu}^{*} L_{\nu}\right\|_{2}^{2}=2 C_{\nu}^{4} \int_{0}^{1} \int_{0}^{x}|c(x, y)|^{2} d y d x=2 C_{\nu}^{4} I_{\nu}^{4} .
$$

Lemma 6.2 For every $\nu \geq 1$,

$$
\frac{\Gamma_{\nu}^{4}}{4 \nu(2 \nu+\alpha+1)} \leq I_{\nu}^{4} \leq \frac{\Gamma_{\nu}^{4}}{8 \nu^{2}}
$$


Proof. The upper bound is obvious because

$$
I_{\nu}^{4}=\frac{1}{2 C_{\nu}^{4}}\left\|L_{\nu}^{*} L_{\nu}\right\|_{2}^{2} \leq \frac{1}{2 C_{\nu}^{4}}\left\|L_{\nu}\right\|_{2}^{4}=\frac{1}{2 C_{\nu}^{4}} \frac{C_{\nu}^{4} \Gamma_{\nu}^{4}}{4 \nu^{2}}=\frac{\Gamma_{\nu}^{4}}{8 \nu^{2}}
$$

due to Lemma 6.1. To obtain the lower bound, note that $x-y s \geq x-x s \geq 0$ for $0 \leq y \leq x$ and $0 \leq s \leq 1$, so that the definition of $I_{\nu}^{4}$ gives

$$
\begin{aligned}
I_{\nu}^{4} & =\int_{0}^{1} x^{-\alpha} \int_{0}^{x} y^{-\alpha}\left(\int_{0}^{y} t^{\alpha}(x-t)^{\nu-1}(y-t)^{\nu-1} d t\right)^{2} d y d x \\
& =\int_{0}^{1} x^{-\alpha} \int_{0}^{x} y^{-\alpha}\left(\int_{0}^{1} y^{\alpha} s^{\alpha}(x-y s)^{\nu-1} y^{\nu-1}(1-s)^{\nu-1} y d s\right)^{2} d y d x \\
& \geq \int_{0}^{1} x^{-\alpha} \int_{0}^{x} y^{-\alpha}\left(\int_{0}^{1} x^{\nu-1} y^{\alpha+\nu} s^{\alpha}(1-s)^{2 \nu-2} d s\right)^{2} d y d x \\
& =\Gamma_{\nu}^{4} \int_{0}^{1} x^{2 \nu-\alpha-2} \int_{0}^{x} y^{\alpha+2 \nu} d y d x=\frac{\Gamma_{\nu}^{4}}{4 \nu(2 \nu+\alpha+1)}
\end{aligned}
$$

We are now in a position to prove Theorem 1.4. From Lemma 6.1 and the inequalities

$$
\frac{\left\|L_{\nu}^{*} L_{\nu}\right\|_{2}^{2}}{\left\|L_{\nu}\right\|_{2}^{2}} \leq\left\|L_{\nu}\right\|_{\infty}^{2} \leq\left\|L_{\nu}\right\|_{2}^{2}
$$

we immediately obtain the estimates

$$
\frac{4 \nu C_{\nu}^{2} I_{\nu}^{4}}{\Gamma_{\nu}^{2}} \leq\left\|L_{\nu}\right\|_{\infty}^{2} \leq \frac{C_{\nu}^{2} \Gamma_{\nu}^{2}}{2 \nu}
$$

and replacing the $I_{\nu}^{4}$ in the lower bound by the lower bound from Lemma 6.2, we arrive at (8). From (8) and (12) we infer that

$$
\left\|L_{\nu}\right\|_{\infty}=\frac{C_{\nu} \Gamma_{\nu}}{\sqrt{2 \nu}}\left(1+O\left(\frac{1}{\nu}\right)\right)=\frac{\sqrt{\Gamma(\alpha+1)}}{2^{1+\alpha / 2}} \frac{1}{\Gamma(\nu) \nu^{1+\alpha / 2}}\left(1+O\left(\frac{1}{\nu}\right)\right),
$$

which implies (9).

To prove Theorem 1.5 we employ the following result.

Proposition 6.3 Let $T$ be a nonzero integral operator of the form $(T f)(x)=$ $\int_{0}^{1} h(x, y) f(y) d y$ on $L^{2}(0,1)$ and suppose $T$ is Hilbert-Schmidt and the kernel $h(x, y)$ assumes only real values. If $\|T\|_{2}^{4} /\left\|T^{*} T\right\|_{2}^{2}=1$ then $\|T\|_{\infty}^{2}=\|T\|_{2}^{2}$, whereas if

$$
k<\frac{\|T\|_{2}^{4}}{\left\|T^{*} T\right\|_{2}^{2}} \leq k+1
$$

for a natural number $k \geq 1$, then

$$
\|T\|_{\infty}^{2} \geq \frac{\|T\|_{2}^{2}}{k+1}+\sqrt{\frac{1}{k(k+1)}\left(\left\|T^{*} T\right\|_{2}^{2}-\frac{\|T\|_{2}^{4}}{k+1}\right)} .
$$


Proof. If $\|T\|_{2}^{4} /\left\|T^{*} T\right\|_{2}^{2}=1$, we have $\|T\|_{2}^{2}=\left\|T^{*} T\right\|_{2} \leq\|T\|_{\infty}\|T\|_{2} \leq\|T\|_{2}^{2}$, which shows that $\|T\|_{\infty}^{2}=\|T\|_{2}^{2}$. So suppose (35) holds for some integer $k \geq 1$. In the case where $\|T\|_{2}^{4} /\left\|T^{*} T\right\|_{2}^{2}=k+1$, inequality (36) amounts to the trivial inequality

$$
\|T\|_{\infty}^{2} \geq \frac{\|T\|_{2}^{2}}{k+1}=\frac{\left\|T^{*} T\right\|_{2}^{2}}{\|T\|_{2}^{2}} .
$$

Thus, assume $\|T\|_{2}^{4} /\left\|T^{*} T\right\|_{2}^{2}<k+1$. Let $\left\{e_{j}\right\}_{j=1}^{\infty}$ be an orthonormal basis of realvalued functions in $L^{2}(0,1)$, e.g., the orthonormal basis formed by cosines and sines, and denote by $P_{N}$ the orthogonal projection of $L^{2}(0,1)$ onto the subspace spanned by $e_{1}, \ldots, e_{N}$. Since $P_{N} T P_{N}$ converges to $T$ both in the operator norm and the Hilbert-Schmidt norm, it suffices to prove that if (35) is satisfied for some real $N \times N$ matrix $T$, then (36) is true for this matrix. But this was shown in [36], [37], [13].

Combining Lemma 6.1 and Proposition 6.3 we arrive after an elementary computation at the lower bound in Theorem 1.5. The upper bound in that theorem results from the inequality $\left\|L_{\nu}\right\|_{\infty}^{2} \leq\left\|L_{\nu}^{*} L_{\nu}\right\|_{2}$ and Lemma 6.1.

We remark that for $\nu=1$ and $\nu=2$ one gets

$$
\begin{aligned}
I_{1}^{4} & =\frac{1}{4} \frac{1}{(\alpha+1)^{2}(\alpha+3)}, \\
I_{2}^{4} & =\frac{1}{4} \frac{5 \alpha^{2}+44 \alpha+99}{(\alpha+1)^{2}(\alpha+2)^{2}(\alpha+3)^{2}(\alpha+5)(\alpha+6)(\alpha+7)} .
\end{aligned}
$$

From Lemmas 6.1 and 6.2 we also see that

$$
\frac{\left\|L_{\nu}\right\|_{2}^{4}}{\left\|L_{\nu}^{*} L_{\nu}\right\|_{2}^{2}}=\frac{\Gamma_{\nu}^{4}}{8 \nu^{2} I_{\nu}^{4}} \leq \frac{2 \nu+\alpha+1}{2 \nu}
$$

does not exceed 2 whenever $\alpha \leq 2 \nu-1$. Consequently, for $-1<\alpha \leq 2 \nu-1$ we may take $k=1$ in Theorem 1.5 .

\section{References}

[1] J. A. Adell and E. A. Gallardo-Gutiérrez: The norm of the Riemann-Liouville operator on $L^{p}[0,1]$ : a probabilistic approach. Bull. Lond. Math. Soc. 39 (2007), 565-574.

[2] J. M. Anderson and A. Hinkkanen: The Cauchy transform on bounded domains. Proc. Amer. Math. Soc. 107 (1989), 179-185.

[3] J. M. Anderson, D. Khavinson, and V. Lomonosov: Spectral properties of some integral operators arising in potential theory. Quart. J. Math. Oxford, Ser. 243 (1992), 387-407. 
[4] P. Borwein and T. Erdélyi: Polynomials and Polynomial Inequalities. Springer-Verlag, New York 1995.

[5] A. Böttcher and P. Dörfler: On the best constants in inequalities of the Markov and Wirtinger types for polynomials on the half-line. Linear Algebra Appl. 430 (2009), 1057-1069.

[6] A. Böttcher and J. Virtanen: Norms of Toeplitz matrices with FisherHartwig symbols. SIAM J. Matrix Anal. Appl. 29 (2007), 660-671.

[7] A. Böttcher and H. Widom: From Toeplitz eigenvalues through Green's kernels to higher-order Wirtinger-Sobolev inequalities. Oper. Theory Adv. Appl. 171 (2007), 73-87.

[8] E. H. Doha: The coefficients of differentiated expansions and derivatives of ultraspherical polynomials. Comput. Math. Appl. 21 (1991), 115-122.

[9] E. H. Doha: On the coefficients of differentiated expansions and derivatives of Jacobi polynomials. J. Phys. A 35 (2002), 3467-3478.

[10] P. Dörfler: New inequalities of Markov type. SIAM J. Math. Anal. 18 (1987), 490-494.

[11] P. Dörfler: A Markov type inequality for higher derivatives of polynomials. Monatshefte f. Math. 109 (1990), 113-122.

[12] P. Dörfler: Über die bestmögliche Konstante in Markov-Ungleichungen mit Laguerre-Gewicht. Österreich. Akad. Wiss. Math.-Natur. Kl. Sitzungsber. II 200 (1991), 13-20.

[13] P. Dörfler: An estimation for the maximum singular value of a matrix. Österreich. Akad. Wiss. Math.-Natur. Kl. Sitzungsber. II 202 (1993), 187194.

[14] P. Dörfler: Asymptotics of the best constant in a certain Markov-type inequality. J. Approx. Theory 114 (2002), 84-97.

[15] M. R. Dostanić: Norm estimate of the Cauchy transform on $L^{p}(\Omega)$. Integral Equations Operator Theory 52 (2005), 465-475.

[16] M. R. Dostanić: The norm and regularized trace of the Cauchy transform. Math. Notes 77 (2005), 777-786.

[17] S. P. Eveson: Norms of iterates of Volterra operators on $L^{2}$. J. Operator Theory 50 (2003), 369-386. 
[18] Z. M. Franco, H. G. Kaper, Man Kam Kwong, and A. Zettl: Best constants in norm inequalities for derivatives on a half-line. Proc. Roy. Soc. Edinburgh Sect. A 100 (1985), 67-84.

[19] N. K. Govil and R.N. Mohapatra: Markov and Bernstein type inequalities for polynomials. J. Inequal. Appl. 3 (1999), 349-387.

[20] P. Halmos: A Hilbert Space Problem Book. D. van Nostrand, Princeton 1967.

[21] E. Hille, G. Szegö, and J. D. Tamarkin: On some generalizations of a theorem of A. Markoff. Duke Math. J. 3 (1937), 729-739.

[22] E. Kamke: Differentialgleichungen. Lösungsmethoden und Lösungen. Teil I: Gewöhnliche Differentialgleichungen. 6. Aufl., Geest \& Portig, Leipzig 1959.

[23] D. Kershaw: Operator norms of powers of the Volterra operator. J. Integral Equations Appl. 11 (1999), 351-362.

[24] A. Kroó: On the exact constant in the $L_{2}$ Markov inequality. J. Approx. Theory 151 (2008), 208-211.

[25] N. Lao and R. Whitley: Norms of powers of the Volterra operator. Integral Equations Operator Theory 27 (1997), 419-425.

[26] G. Little and J. B. Reade: Estimates for the norm of the $n$th indefinite integral. Bull. London Math. Soc. 30 (1998), 539-542.

[27] A. A. Markov: On a question by D. I. Mendeleev. (Russian) Zap. Imp. Akad. St. Petersburg 62 (1890), 1-24.

[28] W. A. Markoff: Über die Funktionen, die in einem gegebenen Intervall möglichst wenig von Null abweichen. Math. Ann. 77 (1916), 213-258.

[29] G. V. Milovanović: An extremal problem for polynomials with nonnegative coefficients. Proc. Amer. Math. Soc. 94 (1985), 423-426.

[30] D. S. Mitrinović, J. E. Pečarić, and A. M. Fink: Inequalities Involving Functions and Their Integrals and Derivatives. Kluwer, Dordrecht 1991.

[31] Q. I. Rahman and G. Schmeisser: Les inégalités de Markoff et de Bernstein. Séminaire de Mathématiques Supérieures 86, Presses de l'Université de Montréal, Montreal 1983.

[32] E. Schmidt: Die asymptotische Bestimmung des Maximums des Integrals über das Quadrat der Ableitung eines normierten Polynoms, dessen Grad ins Unendliche wächst. Sitzungsber. Preuss. Akad. Wiss. (1932), p. 287. 
[33] E. Schmidt: Über die nebst ihren Ableitungen orthogonalen Polynomensysteme und das zugehörige Extremum. Math. Ann. 119 (1944), 165-204.

[34] L. F. Shampine: Some $L_{2}$ Markoff inequalities. J. Res. Nat. Bur. Standards 69B (1965), 155-158.

[35] L. F. Shampine: An inequality of E. Schmidt. Duke Math. J. 33 (1966), $145-150$.

[36] P. Tarazaga: Eigenvalue estimates for symmetric matrices. Linear Algebra Appl. 135 (1990), 171-179.

[37] P. Tarazaga: More estimates for eigenvalues and singular values. Linear Algebra Appl. 149 (1991), 97-110.

[38] B. Thorpe: The norm of powers of the indefinite integral operator on $(0,1)$. Bull. London Math. Soc. 30 (1998), 543-548.

[39] P. Turán: Remark on a theorem of Erhard Schmidt. Mathematica (Cluj) 2 (25) (1960), 373-378.

[40] H. Widom: On the eigenvalues of certain Hermitian operators. Trans. Amer. Math. Soc. 88 (1958), 491-522.

[41] H. Widom: Extreme eigenvalues of translation kernels. Trans. Amer. Math. Soc. 100 (1961), 252-262.

[42] H. Widom: Extreme eigenvalues of $N$-dimensional convolution operators. Trans. Amer. Math. Soc. 106 (1963), 391-414.

Albrecht Böttcher, Fakultät für Mathematik, TU Chemnitz, D-09107 Chemnitz, Germany

aboettch@mathematik.tu-chemnitz.de

Peter Dörfler, Department Mathematik und Informationstechnologie, Montanuniversität Leoben, A-8700 Leoben, Austria

peter.doerfler@mu-leoben.at 